\title{
Early postsurgical diffusivity metrics for prognostication of long-term pain relief after Gamma Knife radiosurgery for trigeminal neuralgia
}

\author{
Sarasa Tohyama, BSc, ${ }^{1-3}$ Peter Shih-Ping Hung, BSc, ${ }^{1-3}$ Jidan Zhong, PhD, ${ }^{1}$ and \\ Mojgan Hodaie, MD ${ }^{1-4}$
}

1Division of Brain, Imaging, and Behaviour-Systems Neuroscience, Krembil Research Institute, Toronto Western Hospital, University Health Network, Toronto; '2Department of Surgery and Institute of Medical Science, Faculty of Medicine, University of Toronto; ${ }^{3}$ Collaborative Program in Neuroscience, University of Toronto; and ${ }^{4}$ Division of Neurosurgery, Krembil Neuroscience Centre, Toronto Western Hospital, University Health Network, Toronto, Ontario, Canada

OBJECTIVE Gamma Knife radiosurgery (GKRS) is an important treatment modality for trigeminal neuralgia (TN). Current longitudinal assessment after GKRS relies primarily on clinical diagnostic measures, which are highly limited in the prediction of long-term clinical benefit. An objective, noninvasive, predictive tool would be of great utility to advance the clinical management of patients. Using diffusion tensor imaging (DTI), the authors' aim was to determine whether early (6 months post-GKRS) target diffusivity metrics can be used to prognosticate long-term pain relief in patients with TN.

METHODS Thirty-seven patients with TN treated with GKRS underwent 3T MRI scans at 6 months posttreatment. Diffusivity metrics of fractional anisotropy, axial diffusivity, radial diffusivity, and mean diffusivity were extracted bilaterally from the radiosurgical target of the affected trigeminal nerve and its contralateral, unaffected nerve. Early (6 months post-GKRS) diffusivity metrics were compared with long-term clinical outcome. Patients were identified as long-term responders if they achieved at least 75\% reduction in preoperative pain for 12 months or longer following GKRS.

RESULTS Trigeminal nerve diffusivity at 6 months post-GKRS was predictive of long-term clinical effectiveness, where long-term responders $(n=19)$ showed significantly lower fractional anisotropy at the radiosurgical target of their affected nerve compared to their contralateral, unaffected nerve and to nonresponders. Radial diffusivity and mean diffusivity, correlates of myelin alterations and inflammation, were also significantly higher in the affected nerve of long-term responders compared to their unaffected nerve. Nonresponders $(n=18)$ did not exhibit any characteristic diffusivity changes after GKRS.

CONCLUSIONS The authors demonstrate that early postsurgical target diffusivity metrics have a translational, clinical value and permit prediction of long-term pain relief in patients with TN treated with GKRS. Importantly, an association was found between the footprint of radiation and clinical effectiveness, where a sufficient level of microstructural change at the radiosurgical target is necessary for long-lasting pain relief. DTI can provide prognostic information that supplements clinical measures, and thus may better guide the postoperative assessment and clinical decision-making for patients with TN. https://thejns.org/doi/abs/10.3171/2018.3.JNS172936

KEYWORDS trigeminal neuralgia; pain; Gamma Knife radiosurgery; diffusion tensor imaging; tractography; neurosurgical prognostication; stereotactic radiosurgery

$\mathrm{S}$ URGICAL prognostication is fundamental to personalized patient care, yet measures that enable prediction of surgical response remain elusive for the chronic pain population. Classic trigeminal neuralgia (TN) is a severe chronic neuropathic facial pain disorder characterized by episodic, electric shock-like attacks of unilateral pain along the divisions of the trigeminal nerve. ${ }^{9}$ Current longitudinal assessment after neurosurgical treatment of TN relies primarily on clinical diagnostic measures, which are highly limited in the prediction of long-term clinical benefit. An objective imaging tool that can be readily applied in a clinical setting would be of great utility, both to better prognosticate pain relief and to facilitate individual pain management.

ABBREVIATIONS AD = axial diffusivity; BNI = Barrow Neurological Institute; $D T I$ = diffusion tensor imaging; FA = fractional anisotropy; FSPGR = fast spoiled gradientrecalled echo; GKRS = Gamma Knife radiosurgery; $M D=$ mean diffusivity; $R D=$ radial diffusivity; $R O I=$ region of interest; $T N=$ trigeminal neuralgia.

SUBMITTED November 21, 2017. ACCEPTED March 19, 2018.

INCLUDE WHEN CITING Published online August 17, 2018; DOI: 10.3171/2018.3.JNS172936. 
Gamma Knife radiosurgery (GKRS) is an important treatment modality for TN that involves delivery of highly focused cobalt- 60 radiation to the cisternal segment of the trigeminal nerve. This neurosurgical procedure, preferred because it is noninvasive, is highly effective, with excellent long-term clinical benefit-some studies suggest that $70 \%-90 \%$ of patients achieve initial pain relief and that more than $50 \%$ of patients remain pain free without medication for as long as 7 years. ${ }^{14,18,22}$ Despite the favorable outcomes, nearly $30 \%$ of patients still experience inadequate relief or recurrence of pain within 24 months of treatment. ${ }^{8,18,22}$ Although all neurosurgical procedures for TN have some variability in response rate, this is especially significant with GKRS, where long-term pain relief is less likely. ${ }^{17,20,28}$

At present, there are no clinical imaging protocols that can determine how effective GKRS has been in the treatment of TN. Current protocols rely on the presence of posttreatment focal $\mathrm{Gd}$ enhancement on the trigeminal nerve in T1-weighted anatomical images to confirm the accuracy of radiation delivery. ${ }^{1}$ However, this enhancement is not seen in every case and is not associated with clinical outcome. ${ }^{19}$ An objective imaging tool that provides clinical value is needed to supplement clinical assessment alone. Such a technique may also be used to predict long-term pain relief. A translational imaging adjunct may allow clinicians to have additional information to rely upon and thus better guide the postoperative assessment and clinical decision-making for patients with TN.

Diffusion tensor imaging (DTI) is an in vivo, noninvasive neuroimaging technique used to measure the movement of water molecules in biological tissue, which permits a thorough characterization of white matter microstructure through quantitative diffusivity metrics. ${ }^{2}$ The most commonly used diffusivity metric, fractional anisotropy (FA), provides insight into the overall white matter microstructural properties, whereas radial diffusivity (RD), axial diffusivity (AD), and mean diffusivity (MD) suggest changes in myelination, ${ }^{26,27}$ axonal integrity, ${ }^{25}$ and underlying neuro-inflammation and edema, ${ }^{2}$ respectively. We have previously shown that there are diffusivity changes in the target region of the trigeminal nerve following GKRS treatment for TN. ${ }^{12}$ To determine whether DTI has a translational, clinical value for the prospective assessment of pain relief, here we assess whether trigeminal nerve diffusivity metrics at a single, early time point after GKRS is predictive of long-term pain relief in patients with TN.

\section{Methods \\ Participants}

This retrospective study approved by the University Health Network Research Ethics Board included 37 patients with TN (23 women, mean age $[ \pm$ SD] $68.3 \pm 13.1$ years) treated at the Toronto Western Hospital in Canada. These patients met the following inclusion criteria: 1) diagnosis of classic, type $1 \mathrm{TN} ; 3,9$ 2) GKRS treatment with no prior surgical procedures for TN; 3) MRI scans at 6 months posttreatment (mean $[ \pm \mathrm{SD}] 6.2 \pm 0.3$ months, range 5-7 months); and 4) at least 12 months of clinical follow-up. Patients with TN secondary to multiple sclero- sis, cranial tumors, or vertebrobasilar dolichoectasia compressing the brainstem were excluded from the study.

\section{Clinical Outcome Assessment}

Clinical outcome measures were obtained through retrospective chart reviews. All patients had at least 12 months of follow-up data. Clinical variables, including medication history, were recorded at every follow-up for each patient, where reported. Pain intensity was measured by the $0-10$ Numeric Rating Scale (10: worst pain imaginable; 0: no pain) and the Barrow Neurological Institute (BNI) scale (class I: no trigeminal pain, no medication; class II: occasional pain, no medication; class III: some pain, adequately controlled with medication; class IV: some pain, not adequately controlled with medication; class V: severe pain, no pain relief). Patients were identified as long-term responders if they achieved at least $75 \%$ reduction in preoperative pain for 12 months or longer following treatment. This cutoff has been previously used as an indicator of excellent surgical response for TN..$^{6,16}$ Patients who did not respond or who experienced early recurrence of pain within 12 months of treatment were classified as nonresponders. Figure 1A depicts the experimental timeline.

\section{Gamma Knife Radiosurgery}

All patients were treated with GKRS using the Leksell Gamma Knife Perfexion unit. The Leksell stereotactic head frame was placed under local anesthesia, and the target was determined based on MR and CT imaging. Using a 4-mm collimator, a single isocenter was positioned at the midcisternal segment of the trigeminal nerve, allowing our constraints to brainstem dosing to remain at less than $15 \mathrm{~Gy}$ to the brainstem $\left(1 \mathrm{~mm}^{3}\right) .80 \mathrm{~Gy}$ was delivered to the $100 \%$ isodose line.

\section{MR Image Acquisition and Processing}

As part of our clinical imaging protocol, each patient underwent 3T GE Signa HDx MRI scans using an 8-channel head coil to obtain the following: T1-weighted fast spoiled gradient-recalled echo (FSPGR) anatomical images (voxel size $0.94 \times 0.94 \times 1 \mathrm{~mm}^{3}$, matrix 256 $\times 256$, TR $12 \mathrm{msec}$, TE $5.1 \mathrm{msec}$, TI $300 \mathrm{msec}$, flip angle $20^{\circ}$, field of view $24 \mathrm{~cm}$ ); contrast-enhanced T1-weighted FLAIR images (voxel size $0.43 \times 0.43 \times 3 \mathrm{~mm}^{3}$, matrix $512 \times 512$, TR $2367 \mathrm{msec}$, TE $13 \mathrm{msec}$, TI $860 \mathrm{msec}$, echo train length 6 , flip angle $90^{\circ}$ ); and diffusion-weighted images (60 directions, spin echo EPI (echo planar imaging) sequence, $1 \mathrm{~B}_{0}, b=1000 \mathrm{sec} / \mathrm{mm}^{2}, 1$ excitation, ASSET (array spatial sensitivity encoding technique), voxel size $0.94 \times 0.94 \times 3 \mathrm{~mm}^{3}$, matrix $256 \times 256$, TR $12 \mathrm{sec}$, TE $86.4 \mathrm{msec}$, flip angle $90^{\circ}$, field of view $24 \mathrm{~cm}$ ).

To analyze the DTI acquisitions, MR images were processed using FSL version 5.0 (FMRIB Software Library, www.fmrib.ox.ac.uk/fsl/) ) $^{24}$ and 3D Slicer version 4.4.0 (NA-MIC, http://www.slicer.org). ${ }^{10}$ Diffusion-weighted images were corrected for eddy-current and motion artifacts in FSL. Subsequently, 3D Slicer was used for DTI estimation, creation of scalar maps (FA, RD, AD, and $M D$ ), visualization of MR images, and linear registration of T1-weighted FSPGR anatomical MR images to diffu- 


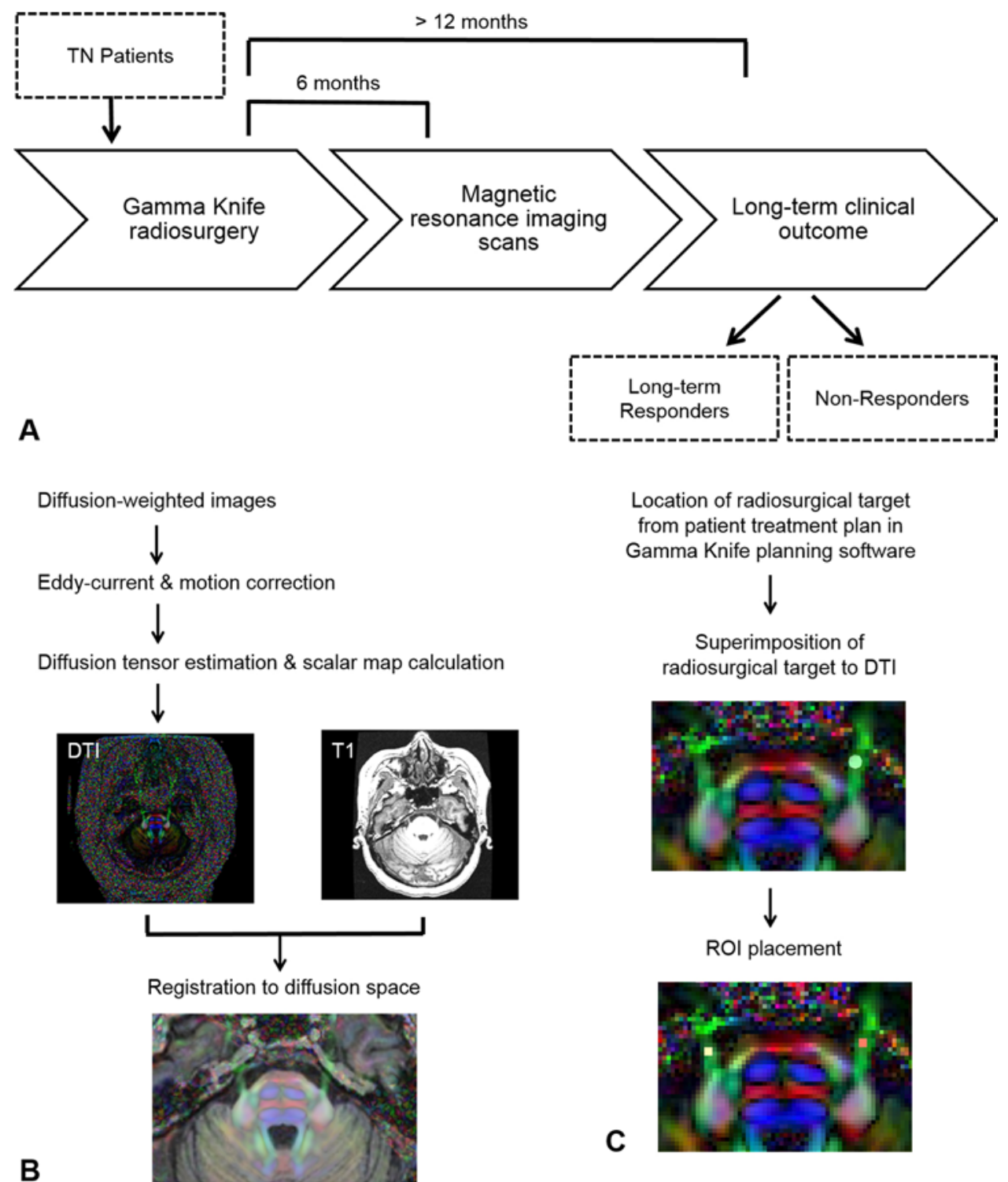

FIG. 1. A: Schematic representation of the experimental timeline. B: Flow diagram of the MRI processing steps: for all patients, diffusion tensor images (upper left) are derived from the diffusion-weighted images. The T1-weighted anatomical images (upper right) are linearly registered to posttreatment diffusion space (lower). All images show the trigeminal nerves in the axial view, emerging from the midpontine level of the brainstem. The DTI is shown in color-by-orientation view (red: left-right; green: anteriorposterior; blue: superior-inferior). C: Flow diagram of process used to define the ROI: for each patient, the radiosurgical target location is determined from the Gamma Knife planning software as a DICOM image, which is superimposed on DTI-an example of a patient with left-sided TN is shown (upper). Masks 4 voxels in size are manually placed at this target ROI in red and the control $\mathrm{ROI}$ is placed in the contralateral, unaffected nerve in yellow (lower). Figure is available in color online only.

sion space for each patient. Figure 1B depicts the MR image processing steps.

\section{Trigeminal Nerve Microstructural Diffusivity Analysis}

For each patient, diffusivity metrics (FA, MD, RD, and $\mathrm{AD})$ were extracted from both the left and right trigeminal nerves at 6 months posttreatment by using 3D Slicer version 4.4.0. ${ }^{10}$ The bilateral nerves were visualized in the axial view at the midpontine level of the brainstem. For the affected nerve, the position of the radiosurgical target was determined based on the location of the target on the patient's treatment plan in the Gamma Knife planning software (GammaPlan, Elekta). This target region of interest (ROI) was defined as 4 voxels in size for all patients, corresponding to the area of the nerve that received approximately $80 \%$ of the radiation dose, including the maximum dose of radiation at $80 \mathrm{~Gy}$. The contralateral, unaffected nerve served as the control. The control ROI was also defined as 4 voxels in size and was positioned in a region mirroring the target ROI. Figure $1 \mathrm{C}$ depicts the steps to ROI placement. These early, 6-month postsurgical diffusivity metrics were compared with long-term clinical outcome. 
To ensure the validity of our method in using the posttreatment unaffected nerve as the control, trigeminal nerve diffusivity metrics were compared between pretreatment and 6 months posttreatment in a subsample of 16 patients who had scans at both time points. Diffusivity metrics were extracted at pretreatment in these patients, with the same ROI definition (i.e., target ROI and control ROI) as described above.

\section{Visual Assessment of the Affected Trigeminal Nerve Using Tractography}

To assess changes in FA at the target region of the trigeminal nerve before versus after GKRS treatment, single tensor tractography was performed for long-term responders and nonresponders. For each patient, both pre- and 6-month posttreatment DTI was acquired and tracts were reconstructed using 3D Slicer version 4.4.0, ${ }^{10}$ with the following parameters: seed spacing $0.3 \mathrm{~mm}$, linear measure start threshold 0.15 , minimum path length $10 \mathrm{~mm}$, FA threshold 0.1, curvature threshold $0.8 \mathrm{rad}$, and integration step length $1 \mathrm{~mm}$.

\section{Evaluation of Treatment Response With Gd Enhancement on the Trigeminal Nerve}

Contrast-enhanced T1-weighted FLAIR anatomical images were examined for the presence of posttreatment Gd enhancement on the treated trigeminal nerve. To confirm that target enhancement does not provide any specific clinical value, the number of long-term responders versus nonresponders with and without Gd enhancement was compared.

\section{Statistical Analysis}

The average microstructural diffusivity differences between the affected and contralateral, unaffected trigeminal nerve for each patient were evaluated using pairedsamples t-tests. Within-group comparisons were also carried out to compare pre- and posttreatment diffusivity metrics by using paired-samples t-tests. The average diffusivity differences among responder and nonresponder groups were evaluated using independent-samples t-tests. The proportion of sex in each group was assessed using the chi-square test. The change in diffusivity values between the affected and unaffected nerve for each individual was expressed in percentage terms: $(\% \Delta D$ DTI Metric $=$ Target - Contra/Contra $\times 100$ ), to reflect the effect of radiation change at the target zone. For the prediction of long-term pain relief, a logistic regression analysis was performed using diffusivity metrics as predictors. Due to multicollinearity, each diffusivity metric (FA, MD, RD, and AD) was included in the model separately, expressed in percentage change terms. All analyses were conducted in SPSS version 20.0 (IBM, Inc.) and statistical significance was determined at $\mathrm{p}<0.05$.

\section{Results}

\section{Patient Demographic and Clinical Characteristics}

Demographic and clinical characteristics of each patient are presented in Table 1. Of the 37 patients with TN, we identified 19 long-term responders (12 women, mean age $[ \pm \mathrm{SD}] 73.8 \pm 10.4$ years) and 18 nonresponders (11 women, mean age $[ \pm \mathrm{SD}] 62.4 \pm 13.3$ years). Long-term responders fit within BNI classes I-III and nonresponders fit within BNI classes IV and V. Responders were significantly older than nonresponders on treatment date $\mathrm{t}_{(35)}=$ $-2.995, p=0.005)$. The distribution of sex in each group was not significantly different $\left(\chi^{2}(1)=0.016, p=0.90\right)$. The mean follow-up period was 34.1 months (range 12.890.4 months). All patients received prior treatment with medications for TN (most commonly carbamazepine) but had inadequate relief and/or side effects.

\section{Trigeminal Nerve Diffusivity Changes After GKRS Are Associated With Long-Term Clinical Effectiveness}

At 6 months post-GKRS, long-term responders showed significantly lower FA at the radiosurgical target of their affected trigeminal nerve compared to their contralateral, unaffected nerve $\left(\mathrm{t}_{(18)}=-3.617, \mathrm{p}=0.002\right)$ and to nonresponders $\left(\mathrm{t}_{(27.316)}=-2.651, \mathrm{p}=0.013\right)$. Nonresponders showed no differences in FA between their affected and unaffected nerve $\left(t_{(17)}=0.686, p=0.50\right)$ (Fig. $2 A$ ). For the specific diffusivities, MD was significantly higher in the affected nerve of responders compared to their unaffected nerve $\left(\mathrm{t}_{(18)}=2.148, \mathrm{p}=0.046\right)$, but did not reach significance compared to nonresponders $\left(\mathrm{t}_{(35)}=1.492, \mathrm{p}=\right.$ 0.15) (Fig. 2B). RD was significantly higher in the affected nerve of responders compared to their unaffected nerve $\left(\mathrm{t}_{(18)}=2.947, \mathrm{p}=0.009\right)$ and trended toward higher $\mathrm{RD}$ compared to nonresponders $\left(\mathrm{t}_{(35)}=1.965, \mathrm{p}=0.057\right)$ (Fig. 2C). No statistically significant differences were observed in $\mathrm{AD}$ that differentiated responders from nonresponders (Fig. 2D). The control, unaffected nerve did not significantly differ between responders and nonresponders for $\mathrm{FA}\left(\mathrm{t}_{(35)}=0.629, \mathrm{p}=0.53\right) ; \mathrm{MD}\left(\mathrm{t}_{(35)}=-1.076, \mathrm{p}=0.29\right) ; \mathrm{RD}$ $\left(\mathrm{t}_{(35)}=-1.128, \mathrm{p}=0.27\right)$; or $\mathrm{AD}\left(\mathrm{t}_{(35)}=-0.885, \mathrm{p}=0.38\right)$.

Comparison of the affected versus unaffected nerve for each individual, expressed as a percentage, also revealed significant differences in FA between responders and nonresponders (Fig. 2E; mean $\pm \mathrm{SEM}_{\text {responders }}=$ $-26.6 \% \pm 7.3 \%$, mean $\pm \mathrm{SEM}_{\text {nonresponders }}=7.0 \% \pm 10.7 \%$; $\left.\mathrm{t}_{(35)}=2.614, \mathrm{p}=0.013\right)$. No statistically significant differences were observed in MD (Fig. 2F; mean $\pm \mathrm{SEM}_{\text {responders }}$ $=28.4 \% \pm 11.9 \%$, mean $\pm \mathrm{SEM}_{\text {nonresponders }}=-1.5 \% \pm 8.5 \%$; $\mathrm{t}_{(35)}=0.572, \mathrm{p}=0.57$ ); RD (Fig. $2 \mathrm{G}$; mean $\pm \mathrm{SEM}_{\text {responders }}=$ $56.5 \% \pm 20.4 \%$, mean $\pm \mathrm{SEM}_{\text {nonresponders }}=-1.4 \% \pm 11.2 \%$; $\mathrm{t}_{(35)}=0.699, \mathrm{p}=0.49$ ); or AD (Fig. 2H; mean $\pm \mathrm{SEM}_{\text {responders }}$ $=7.1 \% \pm 7.1 \%$, mean $\pm \mathrm{SEM}_{\text {nonresponders }}=-2.0 \% \pm 6.3 \% ; \mathrm{t}_{(35)}$ $=0.511, \mathrm{p}=0.61$ ).

\section{Prediction of Long-Term Pain Relief Using Percentage Change in Diffusivity Values}

The logistic regression model for FA was statistically significant $\left(\chi^{2}(1)=7.788, p=0.005\right)$, indicating that FA reliably classified patients into long-term responders and nonresponders for our cohort. The model explained 25.3\% (Nagelkerke $\mathrm{R}^{2}$ ) of the variance in clinical outcome and overall correctly classified $73.0 \%$ of cases. The classification table findings, including sensitivity and specificity, are presented in Table 2. The logistic regression model 
TABLE 1. Demographic and clinical characteristics in 37 patients with TN treated with GKRS

\begin{tabular}{|c|c|c|c|c|c|c|c|}
\hline Case No. & Group & Sex & Age (yrs) & Pain Side & Pain Distribution & Pain Duration (yrs) & Pain Meds \\
\hline 1 & Responder & $\mathrm{F}$ & 78 & Rt & $\mathrm{V} 1 / 2 / 3$ & 7 & GBP \\
\hline 2 & Responder & $\mathrm{F}$ & 86 & $\mathrm{Rt}$ & $\mathrm{V} 2 / 3$ & 10 & CBZ, PGB \\
\hline 3 & Responder & $\mathrm{M}$ & 83 & Rt & V2 & 10 & CBZ \\
\hline 4 & Responder & $\mathrm{F}$ & 77 & $\mathrm{Rt}$ & V3 & 2.5 & None \\
\hline 5 & Responder & M & 76 & Lt & V2 & 4 & CBZ \\
\hline 6 & Responder & $\mathrm{F}$ & 59 & $\mathrm{Rt}$ & $\mathrm{V} 1 / 2 / 3$ & 5 & $\mathrm{CBZ}$ \\
\hline 7 & Responder & $\mathrm{F}$ & 74 & $\mathrm{Lt}$ & $\mathrm{V} 2 / 3$ & NA & CBZ \\
\hline 8 & Responder & M & 87 & Rt & V3 & 12 & $\mathrm{CBZ}$ \\
\hline 9 & Responder & M & 75 & Rt & $\mathrm{V} 2 / 3$ & 12 & CBZ, GBP \\
\hline 10 & Responder & M & 81 & $\mathrm{Lt}$ & $\mathrm{V} 2 / 3$ & 10 & CBZ \\
\hline 11 & Responder & $\mathrm{F}$ & 81 & $\mathrm{Rt}$ & V3 & 20 & CBZ \\
\hline 12 & Responder & $\mathrm{M}$ & 43 & Rt & V3 & 1 & CBZ, PGB \\
\hline 13 & Responder & $\mathrm{F}$ & 76 & $\mathrm{Lt}$ & $V 2 / 3$ & 6 & None \\
\hline 14 & Responder & $\mathrm{F}$ & 73 & $\mathrm{Rt}$ & $\mathrm{V} 1 / 2$ & 16 & $\mathrm{CBZ}$ \\
\hline 15 & Responder & $\mathrm{F}$ & 83 & $\mathrm{Lt}$ & V2 & 11 & GBP \\
\hline 16 & Responder & $\mathrm{F}$ & 68 & $\mathrm{Rt}$ & V2 & 30 & CBZ, GBP \\
\hline 17 & Responder & $\mathrm{F}$ & 70 & $\mathrm{Lt}$ & V3 & 3 & CBZ, GBP \\
\hline 18 & Responder & M & 65 & $\mathrm{Lt}$ & $V 2 / 3$ & 3 & PGB \\
\hline 19 & Responder & $\mathrm{F}$ & 68 & $\mathrm{Lt}$ & $\mathrm{V} 2 / 3$ & 10 & CBZ \\
\hline 20 & Nonresponder & M & 79 & Rt & $\mathrm{V} 2 / 3$ & 20 & CBZ, GBP \\
\hline 21 & Nonresponder & $\mathrm{F}$ & 65 & $\mathrm{Lt}$ & $\mathrm{V} 2 / 3$ & 25 & CBZ \\
\hline 22 & Nonresponder & $\mathrm{F}$ & 49 & Lt & $\mathrm{V} 1 / 2 / 3$ & 5 & CBZ \\
\hline 23 & Nonresponder & $F$ & 75 & $\mathrm{Rt}$ & V2 & 10 & $\mathrm{CBZ}$ \\
\hline 24 & Nonresponder & $\mathrm{F}$ & 61 & Rt & $\mathrm{V} 1 / 2 / 3$ & 2.5 & CBZ, PGB \\
\hline 25 & Nonresponder & $\mathrm{F}$ & 72 & $\mathrm{Lt}$ & V2/3 & 9 & PGB \\
\hline 26 & Nonresponder & $\mathrm{F}$ & 50 & Rt & V3 & 2 & PGB \\
\hline 27 & Nonresponder & M & 60 & $\mathrm{Lt}$ & V3 & 6 & CBZ, GBP \\
\hline 28 & Nonresponder & $\mathrm{F}$ & 55 & $\mathrm{Lt}$ & $\mathrm{V} 1 / 2 / 3$ & 13 & PGB \\
\hline 29 & Nonresponder & $\mathrm{F}$ & 81 & $\mathrm{Lt}$ & V3 & 2.5 & PGB, TOPIR \\
\hline 30 & Nonresponder & M & 67 & Rt & $\mathrm{V} 1 / 2$ & 2 & PGB \\
\hline 31 & Nonresponder & M & 69 & Rt & V3 & 2 & BCF, DLX \\
\hline 32 & Nonresponder & M & 68 & Lt & V3 & 4 & CBZ \\
\hline 33 & Nonresponder & $\mathrm{F}$ & 45 & $\mathrm{Lt}$ & $\mathrm{V} 2 / 3$ & 2 & HYD \\
\hline 34 & Nonresponder & $\mathrm{F}$ & 71 & $\mathrm{Lt}$ & $\mathrm{V} 2 / 3$ & 1.5 & GBP \\
\hline 35 & Nonresponder & $M$ & 76 & Rt & $V 2 / 3$ & 3.5 & GBP, HYD \\
\hline 36 & Nonresponder & M & 33 & $\mathrm{Lt}$ & $\mathrm{V} 1 / 2$ & 2 & CBZ, BCF \\
\hline 37 & Nonresponder & $\mathrm{F}$ & 48 & Rt & $\mathrm{V} 1 / 2 / 3$ & 5 & CBZ, PGB \\
\hline
\end{tabular}

$\mathrm{BCF}=$ baclofen; $\mathrm{CBZ}$ = carbamazepine; $\mathrm{DLX}=$ duloxetine; $\mathrm{GBP}$ = gabapentin; $\mathrm{HYD}=$ hydromorphone; Meds = medications; $\mathrm{NA}=$ information not available; $\mathrm{PGB}=$ pregabalin; TOPIR = topiramate; $\mathrm{V} 1$ = ophthalmic branch of trigeminal nerve; $\mathrm{V} 2$ = maxillary branch; $\mathrm{V} 3=$ mandibular branch.

was not statistically significant for $\operatorname{MD}\left(\chi^{2}(1)=0.345, \mathrm{p}=\right.$ $0.56), \operatorname{RD}\left(\chi^{2}(1)=0.518, \mathrm{p}=0.47\right)$, or $\operatorname{AD}\left(\chi^{2}(1)=0.275, \mathrm{p}\right.$ $=0.60)$ alone, and correctly classified $62.2 \%, 59.5 \%$, and $54.1 \%$ of cases, respectively.

\section{The Contralateral, Unaffected Trigeminal Nerve After GKRS Is a Valid Internal Control}

To ensure that the use of the contralateral, unaffected trigeminal nerve after GKRS is a valid internal control, diffusivity metrics were extracted in a subsample of 16 patients with TN who had both pre- and 6 months posttreatment DTI scans. For FA, the unaffected nerve did not significantly change from pre- to posttreatment in both long-term responders (Fig. 3A; $\mathrm{n}=8$, mean age $[ \pm \mathrm{SD}]$ $68.9 \pm 13.2$ years, $\mathrm{t}_{(7)}=0.267, \mathrm{p}=0.80$ ) and nonresponders (Fig. 3B; $\mathrm{n}=8$, mean age $[ \pm \mathrm{SD}] 62.6 \pm 15.0$ years, $\mathrm{t}_{(7)}=$ $-0.819, p=0.44)$. In the affected nerve, responders showed significantly lower FA from pre- to posttreatment $\left(\mathrm{t}_{(7)}=\right.$ $-2.887, \mathrm{p}=0.023$ ), whereas nonresponders showed no statistical difference $\left(\mathrm{t}_{(7)}=0.657, \mathrm{p}=0.53\right)$. Additionally, responders showed significantly lower FA in their affect- 

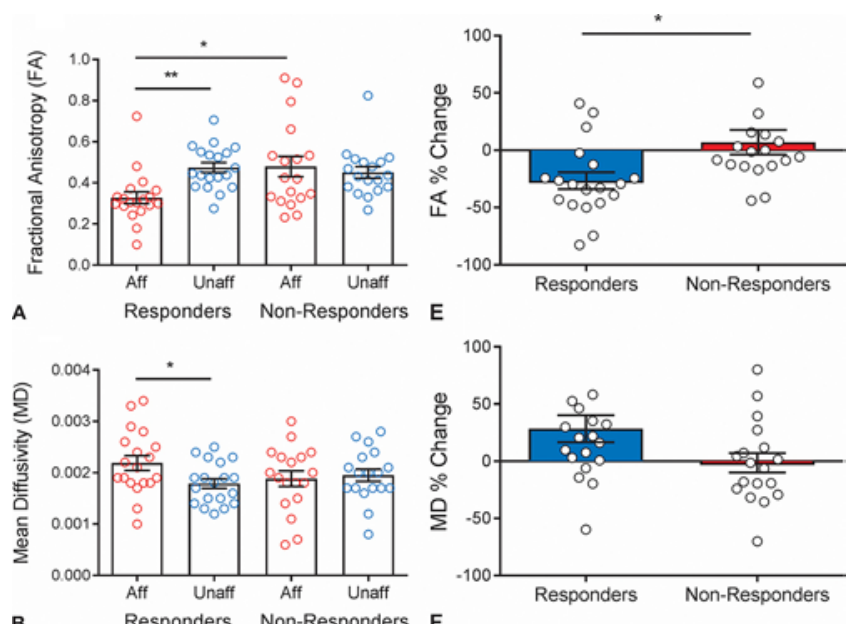

B
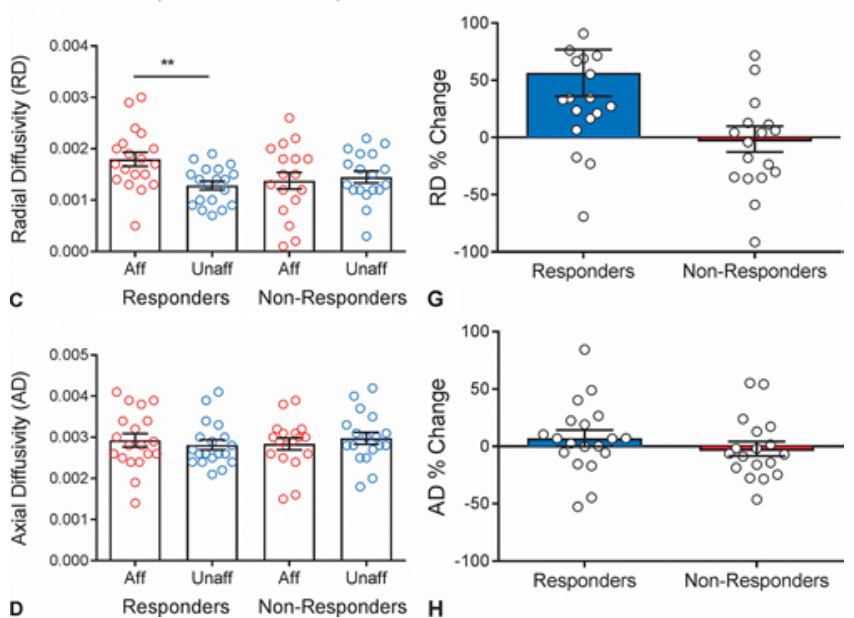

FIG. 2. Diffusivity metrics taken at 6 months post-GKRS. A-D: Bar graphs showing the average DTI-derived values \pm SEM with overlaid individual patient data for the affected (red) and unaffected (blue) trigeminal nerve in long-term responders and nonresponders. Responders show characteristically lower $F A(A)$, higher MD $(B)$, higher $R D(C)$, and no change in $A D(D)$ in their affected nerve compared to their unaffected nerve. Compared to nonresponders, responders also show distinctively lower FA (red bars, panel A) and a trend toward higher RD ( $p=0.057)$ (red bars, panel $\mathrm{C}$ ) in their affected nerve. E-H: Bar graphs showing the DTI-derived values computed as a percentage change for each individual: \% $\%$ DTI Metric = Target - Contra/Contra $\times 100( \pm$ SEM) for long-term responders (blue) and nonresponders (red). FA differentiates responders from nonresponders $(E)$, whereas $M D, R D$, and $A D$ do not $(\mathrm{F}-\mathrm{H})$. . Aff $=$ affected nerve; Unaff $=$ unaffected nerve. ${ }^{*} p<0.05,{ }^{* *} p<$ 0.01 . Figure is available in color online only.

ed nerve compared to their unaffected nerve at 6 months posttreatment $\left(\mathrm{t}_{(7)}=-3.658, \mathrm{p}=0.008\right)$, whereas nonresponders showed no differences $\left(\mathrm{t}_{(7)}=-0.377, \mathrm{p}=0.72\right)$. Specific diffusivities (MD, RD, and AD) of the affected and unaffected trigeminal nerve at both pre- and 6 months posttreatment for long-term responders and nonresponders are shown in Table 3. The unaffected nerve did not significantly change from pre- to posttreatment in both responders and nonresponders for MD, RD, and AD. Furthermore, responders showed a significantly higher RD from pre- to posttreatment in the affected nerve $\left(\mathrm{t}_{(7)}=2.452, \mathrm{p}=0.044\right)$ as well as between the affected and unaffected nerve at 6
TABLE 2. Classification table resulting from logistic regression model predicting clinical outcome from FA

\begin{tabular}{lccc}
\hline \multicolumn{1}{c}{ Group } & $\begin{array}{c}\text { Correct } \\
\text { Prediction }\end{array}$ & $\begin{array}{c}\text { Incorrect } \\
\text { Prediction }\end{array}$ & $\begin{array}{c}\text { Percentage } \\
\text { Correct }\end{array}$ \\
\hline Responders & 15 & 4 & 78.9 \\
\hline Nonresponders & 12 & 6 & 66.7 \\
\hline Overall & 27 & 10 & 73.0 \\
\hline
\end{tabular}

months posttreatment $\left(\mathrm{t}_{(7)}=2.832, \mathrm{p}=0.025\right)$. No statistically significant differences were observed for MD or AD.

\section{Tractography Illustrates Microstructural Changes in the Affected Trigeminal Nerve After GKRS}

Figure 4 depicts the affected trigeminal nerve tracts, superimposed on a DTI/T1-weighted anatomical image. The change in FA after GKRS is visualized at the radiosurgical target in the long-term responder (Fig. 4A and B), but not in the nonresponder (Fig. 4C and D). The grouplevel diffusivity metrics are also measured from this target region of the nerve.

\section{Gd Enhancement After GKRS Is Not Associated With Clinical Outcome}

Of the 32 patients with TN in whom contrast-enhanced T1-weighted FLAIR images were obtained, 19 patients exhibited focal Gd enhancement on the treated trigeminal nerve. Of these, 10 were long-term responders and 9 were nonresponders. Among the 13 patients who did not exhibit enhancement, 6 were long-term responders and 7 were nonresponders.

\section{Discussion}

We demonstrate for the first time that target diffusivity metrics at 6 months posttreatment can predict long-term pain relief in patients with TN treated with GKRS. Specifically, long-term responders showed a characteristically lower FA, higher RD, and higher MD at the radiosurgical target of their affected nerve compared to their control, unaffected nerve. The changes are sufficiently different to allow for this feature to be directly translated into the clinical follow-up of patients. Moreover, our findings reflect the possibility of a threshold effect where, based on the degree of microstructural change in the nerve, we can determine whether there will be long-term clinical benefit. We attempted to select a practical timeline that corresponds to the typical follow-up for these patients. For this reason, we examined 6-month posttreatment MRI and clinical follow-up of at least 12 months for long-term assessment. We consider 6 months posttreatment to be an early time point because it is a common clinical and imaging follow-up time frame.

\section{Microstructural Diffusivities Differentiating Long-Term Responders From Nonresponders to GKRS}

Among the different diffusivity metrics, FA appeared to be the best prognostic indicator for long-term pain re- 

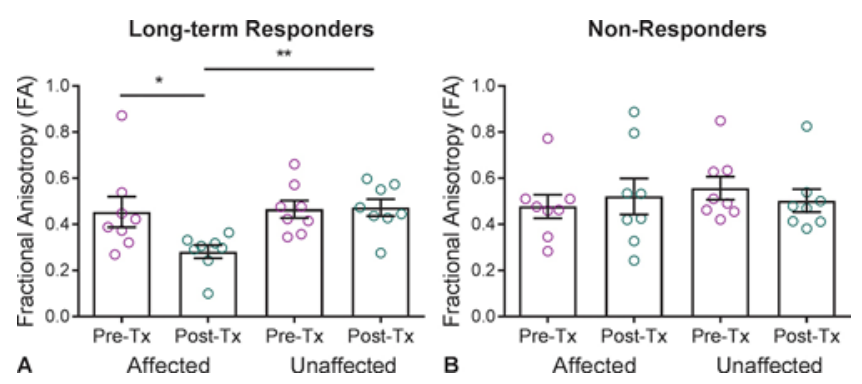

FIG. 3. Bar graphs showing the average FA values \pm SEM with overlaid individual patient data at pretreatment (purple) and 6 months posttreatment (green) in the affected and unaffected trigeminal nerve of longterm responders $(\mathbf{A})$ and nonresponders $(\mathbf{B})$. Long-term responders show a substantial decrease in posttreatment FA in their affected nerve. These changes are not observed in nonresponders. The unaffected nerve remains unchanged between pre- and posttreatment for responders and nonresponders. Tx $=$ treatment. ${ }^{*} p<0.05,{ }^{* *} p<0.01$. Figure is available in color online only.

lief, where long-term responders showed a significant decrease in FA at the radiosurgical target of their affected nerve compared to their contralateral, unaffected nerve and to nonresponders. Nonresponders did not show any characteristic changes in FA. Our findings suggest that for patients to achieve long-lasting pain relief, sufficient microstructural changes at the radiosurgical target must be present at early follow-up. Furthermore, logistic regression analysis suggests the possibility of a threshold effect, whereby quantifying the degree of microstructural change allows us to predict on an individual level whether there will be long-lasting pain relief. For our cohort of patients, long-term responders showed on average a $26.6 \%$ decrease in FA, whereas nonresponders exhibited a $7 \%$ increase. This implies that in our group, an approximately $30 \%$ decrease in target zone FA is predictive of long-term clinical benefit after GKRS.

Because GKRS has highly accurate targeting, ${ }^{19}$ individual variations in the effectiveness of radiosurgery are more likely to be due to the range of biological responses to radiation. ${ }^{21}$ Although this has been demonstrated in tumors before, ${ }^{21}$ it is less studied in single-isocenter treatments such as GKRS for TN. Therefore, our findings provide important insight into how DTI appears to be a viable in vivo model through which the effects of radiation on a nerve can be assessed. As a result, DTI may serve as an important histopathological correlate.

Although studies report that 70\%-90\% of patients with TN achieve adequate pain relief from GKRS, ${ }^{14,18,22}$ our cohort was composed of a roughly equal split between those who benefited from treatment and nonresponders. This discrepancy is related to the inherent selection bias that is required for our inclusion criteria, such as absence of prior surgical procedures for TN, availability of 6-month DTI scans, and at least 12 months of clinical assessment. Furthermore, it is likely that patients who have a suboptimal response will have ongoing clinical and MR assessment.

FA change has been shown to be indicative of numerous pathologies, ${ }^{5}$ including TN. ${ }^{7,11,15}$ Although FA provides insight into the overall white matter microstructure, it does not offer details into the specific types of microstructural
TABLE 3. Specific trigeminal nerve diffusivity metrics in a subsample of 16 patients with pre- and posttreatment DTI scans

\begin{tabular}{|c|c|c|c|}
\hline Group & Comparison & Diffusivity & t Value \\
\hline \multirow{9}{*}{ Responders } & \multirow{3}{*}{$\begin{array}{l}\text { Pre- vs post-GKRS, } \\
\text { affected nerve }\end{array}$} & MD & 1.648 \\
\hline & & $\mathrm{RD}^{*}$ & 2.452 \\
\hline & & $A D$ & 0.557 \\
\hline & \multirow{3}{*}{$\begin{array}{l}\text { Pre- vs post-GKRS, } \\
\text { unaffected nerve }\end{array}$} & MD & 1.350 \\
\hline & & $\mathrm{RD}$ & 0.981 \\
\hline & & $A D$ & 1.803 \\
\hline & \multirow{3}{*}{$\begin{array}{l}\text { Post-GKRS, affected vs } \\
\text { unaffected nerve }\end{array}$} & MD & 1.575 \\
\hline & & $\mathrm{RD}^{*}$ & 2.832 \\
\hline & & $A D$ & -0.456 \\
\hline \multirow{9}{*}{ Nonresponders } & \multirow{3}{*}{$\begin{array}{l}\text { Pre- vs post-GKRS, } \\
\text { affected nerve }\end{array}$} & MD & 0.635 \\
\hline & & $\mathrm{RD}$ & 0.395 \\
\hline & & $A D$ & 0.937 \\
\hline & \multirow{3}{*}{$\begin{array}{l}\text { Pre- vs post-GKRS, } \\
\text { unaffected nerve }\end{array}$} & MD & 0.018 \\
\hline & & $\mathrm{RD}$ & 0.199 \\
\hline & & $A D$ & -0.224 \\
\hline & \multirow{3}{*}{$\begin{array}{l}\text { Post-GKRS, affected vs } \\
\text { unaffected nerve }\end{array}$} & $M D$ & 0.432 \\
\hline & & $\mathrm{RD}$ & 0.423 \\
\hline & & $A D$ & 0.419 \\
\hline
\end{tabular}

Degrees of freedom $=7$ for all t-tests.

${ }^{*} p<0.05$.

changes. Therefore, by examining RD, AD, and MD, we can gain further understanding of changes in myelination, ${ }^{26,27}$ axonal integrity, ${ }^{25}$ and possible underlying neuroinflammation and edema ${ }^{2}$ that are integral to the overall microstructural organization and alterations that result after radiation delivery.

Examination of these diffusivity metrics revealed RD to show noteworthy changes. Specifically, long-term responders showed significantly higher RD in their affected nerve compared to their unaffected nerve, whereas AD remained unaltered. Because MD takes the average of RD and $\mathrm{AD}$, long-term responders also showed significantly higher MD in their affected nerve compared to their unaffected nerve, suggesting possible underlying neuroinflammation and edema. ${ }^{2}$ These findings show that the changes seen in composite parameter FA are largely driven by RD changes, which suggests that the microstructural alterations present in long-term responders may primarily be influenced by the effect of radiation on myelin as opposed to axonal damage. This result is consistent with our clinical observation of lack of sensory deficits after GKRS in this group. Previous studies have also reported a low incidence of troublesome sensory changes after GKRS in patients with TN. ${ }^{8,22}$

\section{DTI as a Tool for Neurosurgical Prognostication}

Because DTI provides an in vivo, noninvasive means to quantitatively assess radiation effects on tissue microstructure, it is a clinically feasible tool for this type of neurosurgical prognostication. At present, longitudinal assessment after GKRS for patients with TN consists of ongoing, clinical follow-up approximately every 6 months. 

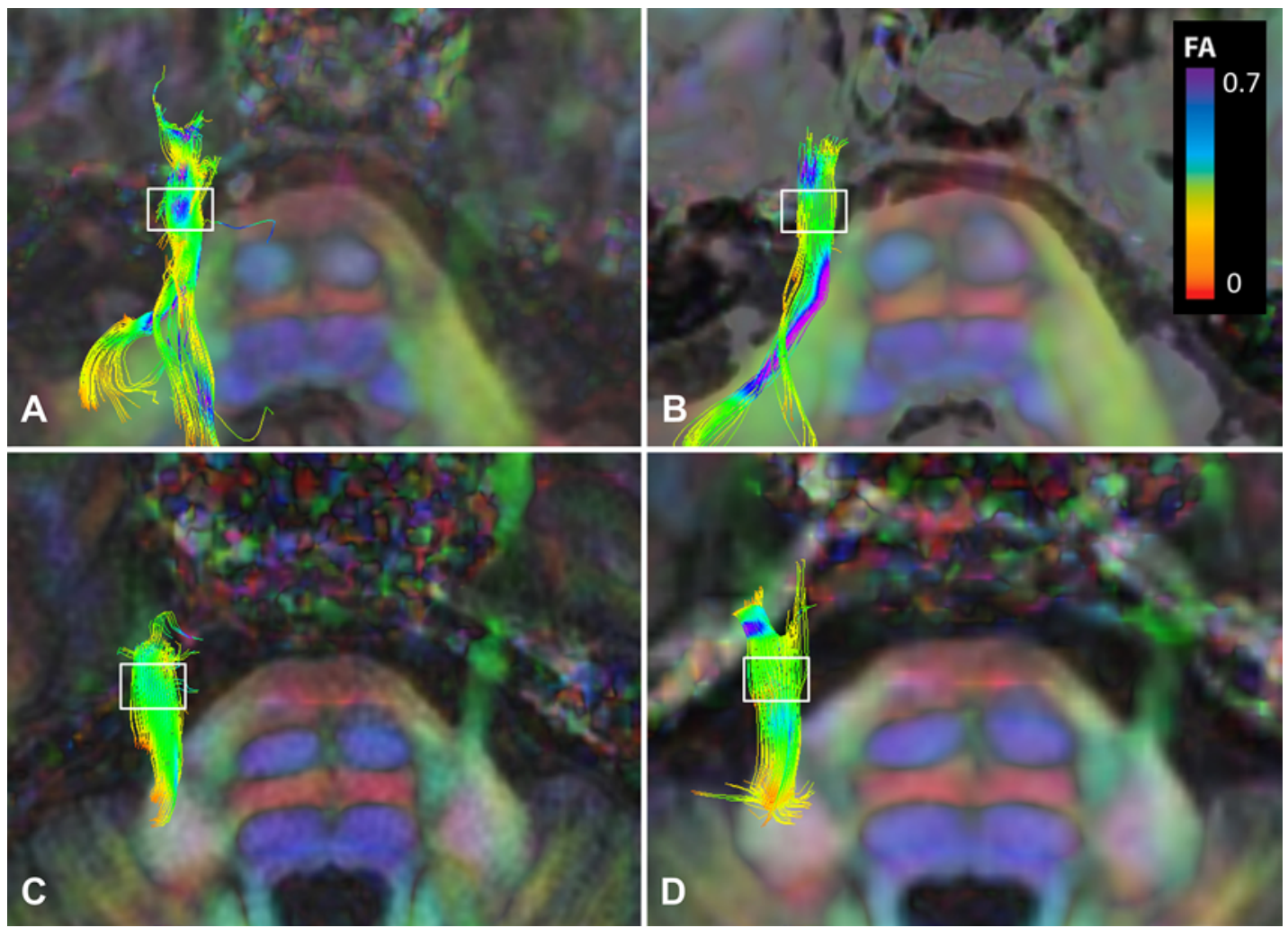

FIG. 4. Visual representation of the affected trigeminal nerve using tractography. The reconstructed tracts are overlaid onto the axial DTI/T1-weighted anatomical images at the midpontine level of the brainstem. The colors of the tracts represent the spectrum values of FA ( 0 to 0.7 ), as shown by the legend at the top right corner. The white box denotes the target area of the nerve where the radiation was delivered. The top 2 images $(\mathbf{A}$ and $\mathbf{B})$ correspond to a long-term responder (single subject). Distinct changes in FA are visible at the target area when compared from pretreatment $(A)$ to 6 months posttreatment $(B)$, as represented by a shift in color from the purple/blue spectrum to green/yellow. The bottom 2 images (C and $\mathbf{D})$ correspond to a nonresponder. In this patient, no FA changes are observed at the target area between pretreatment (C) and 6 months posttreatment (D). Figure is available in color online only.

As such, the current postoperative assessment relies solely on clinical measures, which does not permit prediction of long-term pain relief. By timely integration of DTI posttreatment, clinicians can draw on additional information and may better assess the long-term outcome of patients in the clinic. Figure 5 illustrates the current postoperative assessment in comparison to the postoperative assessment that incorporates our prognostic approach-we anticipate the integration of DTI assessment into the postoperative management of patients with TN who undergo GKRS. The present study shows DTI to have a translational, clinical value in the assessment of TN treatment outcome.

We also show that the contralateral, unaffected trigeminal nerve after GKRS is a valid internal control. Comparison of the microstructural properties of the unaffected nerve pre- versus 6 months posttreatment did not reveal any significant diffusivity changes for both long-term responders and nonresponders. We also previously reported that there are no significant diffusivity changes in the cisternal segment of the unaffected trigeminal nerve between patients with TN and healthy controls. ${ }^{4}$ This implies that MRI scans do not need to be acquired at multiple time points and that the use of 6-month DTI alone is practical.

Furthermore, examination of the trigeminal nerve before treatment did not show any characteristic signature that differentiates long-term responders from nonresponders, suggesting that prediction of treatment response may not be feasible prior to GKRS treatment. In our previous study, we found presurgical microstructural abnormalities in the pontine segment that are characteristic of TN nonresponders. ${ }^{13}$ The important distinction between our previous study and the current one is that earlier we examined multiple anatomical sites along the trigeminal nerve, such as the root entry zone and pontine segment, as well as response to both GKRS and microvascular decompression surgery. In the current study, we find that the role of sufficient microstructural changes in the nerve, specifically at the radiosurgical target, is what is critical for GKRS effectiveness. Although multiple factors work together in predicting treatment response, both studies demonstrate that DTI is a strong, objective imaging tool for neurosurgical prognostication that can improve the clinical management of patients.

\section{Limitations of the Study}

The current scope of this study involves the use of 3T MRI with 60-directions DTI, and expertise to perform the appropriate DTI analyses. At present, our approach does not consist of a fully automated pipeline, because regis- 


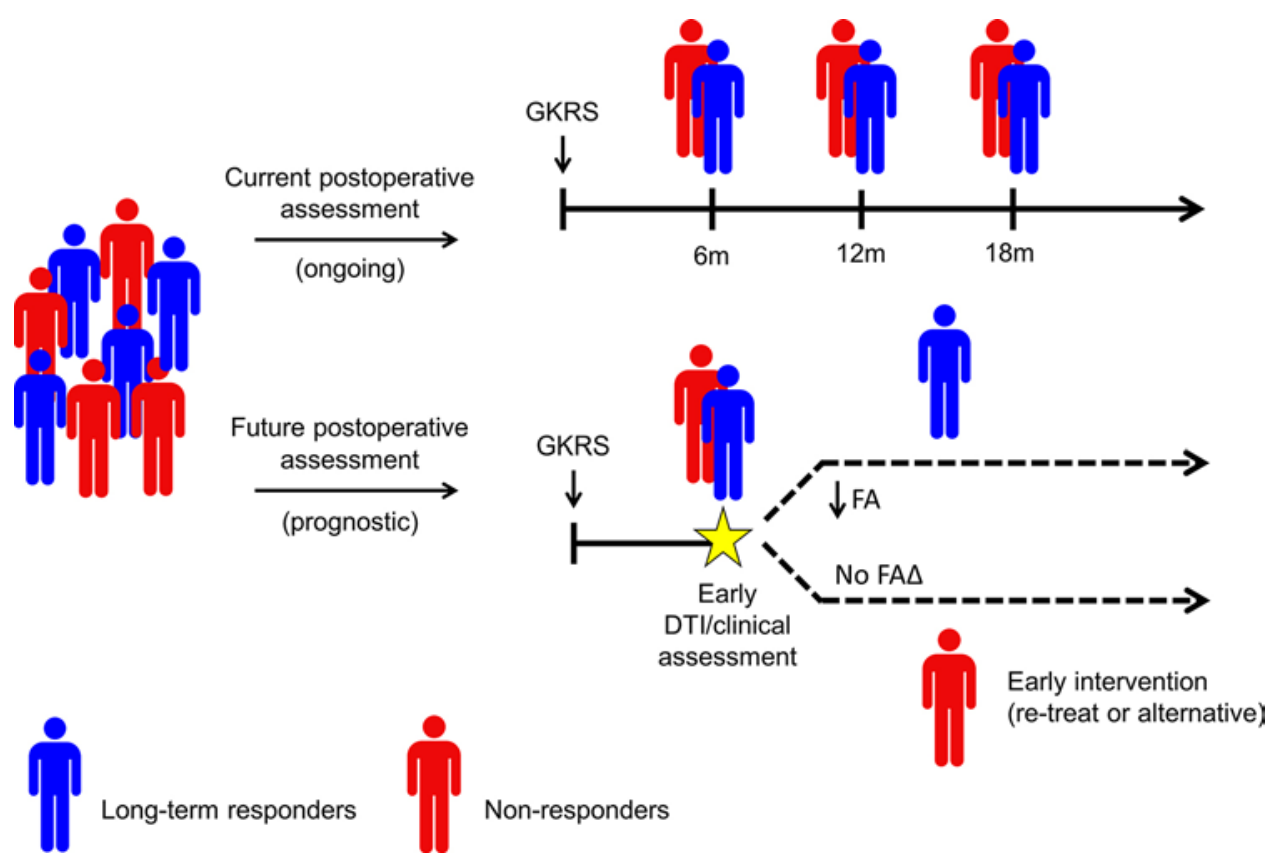

FIG. 5. Schematic representation comparing the current postoperative assessment, which relies solely on ongoing clinical followup (upper), with the expected postoperative assessment proposed in our study (lower). Our prognostic approach incorporates early DTI assessment with clinical measures. Patients who experience long-lasting pain relief after GKRS show a significant decrease in target zone FA, whereas patients who do not experience adequate pain relief show marginal changes in FA. This suggests that DTI metrics may help in earlier clinical decision-making for those who do not show improvement in pain following GKRS treatment. $m=$ months. Figure is available in color online only.

tration and ROI placements must be performed manually. There are important elements to consider when integrating DTI into clinical practice, including imaging coregistration and assessment of accuracy, which we have previously discussed in detail..$^{23}$ The individual anatomical variability and size of the trigeminal nerve across patients must also be noted. This is important in the evaluation and assessment of the target area and contralateral control. Despite these limitations, the present study is one of a growing number of investigations that show DTI to have a translational, clinical value that aids neurosurgeons in treatment planning and in defining surgical targets, and it offers the promise of improving clinical outcome.

\section{Conclusions}

In an era of precision medicine, the application of DTI as a prognostic tool for personalized clinical decisionmaking for patients with chronic pain holds important promise. DTI appears to be a strong, objective measure to assess the effects of radiation on a nerve. Moreover, assessment of the trigeminal nerve using DTI at early (6-month) follow-up predicts long-term pain relief in patients with TN who are treated with GKRS. Importantly, there seems to be a need for sufficient microstructural changes at the radiosurgical target for patients to achieve long-lasting pan relief. Incorporating early DTI assessment can provide prognostic information that supplements clinical measures, and thus better guide the postoperative assessment and clinical decision-making for patients with TN. Because our study was retrospective, future studies should conduct a prospective trial and apply this approach to a new, larger patient population.

\section{Acknowledgments}

We thank Erika Wharton-Shukster and Jia-Yan Zhang for assistance with chart reviews and Powell Chu for technical assistance. This study is supported by the Canadian Institutes of Health Research operating grant (Ref. \# MOP130555) and the Trigeminal Neuralgia Association of Canada. Ms. Tohyama is the recipient of the University of Toronto Centre for the Study of Pain Scientist Scholarship.

\section{References}

1. Alberico RA, Fenstermaker RA, Lobel J: Focal enhancement of cranial nerve $\mathrm{V}$ after radiosurgery with the Leksell gamma knife: experience in 15 patients with medically refractory trigeminal neuralgia. AJNR Am J Neuroradiol 22:1944-1948, 2001

2. Alexander AL, Lee JE, Lazar M, Field AS: Diffusion tensor imaging of the brain. Neurotherapeutics 4:316-329, 2007

3. Burchiel KJ: A new classification for facial pain. Neurosurgery 53:1164-1167, 2003

4. Chen DQ, DeSouza DD, Hayes DJ, Davis KD, O'Connor P, Hodaie M: Diffusivity signatures characterize trigeminal neuralgia associated with multiple sclerosis. Mult Scler 22:51-63, 2016

5. Ciccarelli O, Catani M, Johansen-Berg H, Clark C, Thompson A: Diffusion-based tractography in neurological disorders: concepts, applications, and future developments. Lancet Neurol 7:715-727, 2008

6. DeSouza DD, Davis KD, Hodaie M: Reversal of insular and microstructural nerve abnormalities following effective sur- 
gical treatment for trigeminal neuralgia. Pain 156:1112-1123, 2015

7. DeSouza DD, Hodaie M, Davis KD: Abnormal trigeminal nerve microstructure and brain white matter in idiopathic trigeminal neuralgia. Pain 155:37-44, 2014

8. Dhople AA, Adams JR, Maggio WW, Naqvi SA, Regine WF, Kwok Y: Long-term outcomes of Gamma Knife radiosurgery for classic trigeminal neuralgia: implications of treatment and critical review of the literature. Clinical article. J Neurosurg 111:351-358, 2009

9. Eller JL, Raslan AM, Burchiel KJ: Trigeminal neuralgia: definition and classification. Neurosurg Focus 18(5):E3, 2005

10. Fedorov A, Beichel R, Kalpathy-Cramer J, Finet J, FillionRobin JC, Pujol S, et al: 3D Slicer as an image computing platform for the Quantitative Imaging Network. Magn Reson Imaging 30:1323-1341, 2012

11. Herweh C, Kress B, Rasche D, Tronnier V, Tröger J, Sartor $\mathrm{K}$, et al: Loss of anisotropy in trigeminal neuralgia revealed by diffusion tensor imaging. Neurology 68:776-778, 2007

12. Hodaie M, Chen DQ, Quan J, Laperriere N: Tractography delineates microstructural changes in the trigeminal nerve after focal radiosurgery for trigeminal neuralgia. PLoS One 7:e32745, 2012

13. Hung PSP, Chen DQ, Davis KD, Zhong J, Hodaie M: Predicting pain relief: Use of pre-surgical trigeminal nerve diffusion metrics in trigeminal neuralgia. Neuroimage Clin 15:710718,2017

14. Kondziolka D, Zorro O, Lobato-Polo J, Kano H, Flannery TJ, Flickinger JC, et al: Gamma Knife stereotactic radiosurgery for idiopathic trigeminal neuralgia. J Neurosurg 112:758765,2010

15. Leal PRL, Roch JA, Hermier M, Souza MAN, Cristino-Filho G, Sindou M: Structural abnormalities of the trigeminal root revealed by diffusion tensor imaging in patients with trigeminal neuralgia caused by neurovascular compression: a prospective, double-blind, controlled study. Pain 152:23572364, 2011

16. Li ST, Pan Q, Liu N, Shen F, Liu Z, Guan Y: Trigeminal neuralgia: what are the important factors for good operative outcomes with microvascular decompression. Surg Neurol 62:400-405, 2004

17. Linskey ME, Ratanatharathorn V, Peñagaricano J: A prospective cohort study of microvascular decompression and Gamma Knife surgery in patients with trigeminal neuralgia. J Neurosurg 109 Suppl:160-172, 2008

18. Martínez Moreno NE, Gutiérrez-Sárraga J, Rey-Portolés G, Jiménez-Huete A, Martínez Álvarez R: Long-term outcomes in the treatment of classical trigeminal neuralgia by Gamma Knife radiosurgery: a retrospective study in patients with minimum 2-year follow-up. Neurosurgery 79:879-888, 2016

19. Massager N, Abeloos L, Devriendt D, Op de Beeck M, Levivier M: Clinical evaluation of targeting accuracy of gamma knife radiosurgery in trigeminal neuralgia. Int J Radiat Oncol Biol Phys 69:1514-1520, 2007

20. Mendelson ZS, Velagala JR, Kohli G, Heir GM, Mammis A, Liu JK: Pain-free outcomes and durability of surgical intervention for trigeminal neuralgia: a comparison of Gamma Knife and microvascular decompression. World Neurosurg 112:e732-e746, 2018

21. Niranjan A, Gobbel GT, Kondziolka D, Flickinger JC, Lunsford LD: Experimental radiobiological investigations into radiosurgery: present understanding and future directions. Neurosurgery 55:495-505, 2004

22. Régis J, Tuleasca C, Resseguier N, Carron R, Donnet A, Gaudart J, et al: Long-term safety and efficacy of Gamma Knife surgery in classical trigeminal neuralgia: a 497-patient historical cohort study. J Neurosurg 124:1079-1087, 2016

23. Sammartino F, Rowland N, Hodaie M, Kalia SK, Lozano AM, Hamani C: Diffusion tensor imaging and deep brain stimulation. Expert Rev Med Devices 13:615-617, 2016

24. Smith SM, Jenkinson M, Woolrich MW, Beckmann CF, Behrens $\mathrm{TE}$, Johansen-Berg $\mathrm{H}$, et al: Advances in functional and structural MR image analysis and implementation as FSL. Neuroimage 23 (Suppl 1):S208-S219, 2004

25. Song SK, Sun SW, Ju WK, Lin SJ, Cross AH, Neufeld AH: Diffusion tensor imaging detects and differentiates axon and myelin degeneration in mouse optic nerve after retinal ischemia. Neuroimage 20:1714-1722, 2003

26. Song SK, Sun SW, Ramsbottom MJ, Chang C, Russell J, Cross AH: Dysmyelination revealed through MRI as increased radial (but unchanged axial) diffusion of water. Neuroimage 17:1429-1436, 2002

27. Song SK, Yoshino J, Le TQ, Lin SJ, Sun SW, Cross AH, et al: Demyelination increases radial diffusivity in corpus callosum of mouse brain. Neuroimage 26:132-140, 2005

28. Wang DD, Raygor KP, Cage TA, Ward MM, Westcott S, Barbaro NM, et al: Prospective comparison of long-term pain relief rates after first-time microvascular decompression and stereotactic radiosurgery for trigeminal neuralgia. J Neurosurg 128: $68-77,2018$

\section{Disclosures}

The authors report no conflict of interest concerning the materials or methods used in this study or the findings specified in this paper.

\section{Author Contributions}

Conception and design: Hodaie, Tohyama. Acquisition of data: Tohyama, Hung. Analysis and interpretation of data: all authors. Drafting the article: Tohyama. Critically revising the article: all authors. Reviewed submitted version of manuscript: all authors. Approved the final version of the manuscript on behalf of all authors: Hodaie. Statistical analysis: Tohyama. Study supervision: Hodaie.

\section{Supplemental Information \\ Previous Presentations}

Portions of this work were presented in abstract form at the World Society for Stereotactic and Functional Neurosurgery Congress, Berlin, Germany, June 28, 2017; Canadian Pain Society Annual Scientific Meeting, Halifax, Nova Scotia, Canada, May 25, 2017; and the Society for Neuroscience Annual Meeting, Washington, DC, November 15, 2017.

\section{Correspondence}

Mojgan Hodaie: Toronto Western Hospital, Toronto, ON, Canada. mojgan.hodaie@uhn.ca. 